\title{
FAST CHANGES IN DIRECTION DURING HUMAN LOCOMOTION ARE EXECUTED BY IMPULSIVE ACTIVATION OF MOTOR MODULES
}

\author{
A. S. OLIVEIRA, ${ }^{a, b}$ P. B. SILVA, ${ }^{a}$ M. E. LUND, ${ }^{c}$ \\ U. G. KERSTING ${ }^{a}$ AND D. FARINA ${ }^{\mathrm{d} *}$ \\ ${ }^{\text {a } C e n t e r ~ f o r ~ S e n s o r y-M o t o r ~ I n t e r a c t i o n, ~ D e p a r t m e n t ~ o f ~ H e a l t h ~}$ \\ Science and Technology, Aalborg University, Aalborg, Denmark \\ b The CAPES Foundation, Brazilian Education Ministry, \\ Brasilia, Brazil \\ ${ }^{\mathrm{c}}$ Department of Mechanical and Manufacturing Engineering, Aalborg \\ University, Aalborg, Denmark \\ ${ }^{\mathrm{d}}$ Department of Neurorehabilitation Engineering, Bernstein Focus \\ Neurotechnology Göttingen, Bernstein Center for \\ Computational Neuroscience, University Medical Center Göttingen, \\ Georg-August University, Göttingen, Germany
}

\begin{abstract}
This study investigated the modular control of complex locomotor tasks that require fast changes in direction, i.e., cutting manoeuvres. It was hypothesized that such tasks are accomplished by an impulsive (burst-like) activation of a few motor modules, as observed during walking and running. It was further hypothesized that the performance in cutting manoeuvres would be associated to the relative timing of the activation impulses. Twenty-two healthy men performed $90^{\circ}$ side-step cutting manoeuvres while electromyography (EMG) activity from 16 muscles of the supporting limb and trunk, kinematics, and ground reaction forces were recorded. Motor modules and their respective temporal activations were extracted from the EMG signals by non-negative matrix factorization. The kinematic analysis provided the velocity of the centre of mass and the external work absorbed during the load acceptance (negative work, external work during absorption (W-Abs)) and propulsion phases (positive work, external work during propulsion (W-Prp)) of the cutting manoeuvres. Five motor modules explained the EMG activity of all muscles and were driven in an impulsive way, with timing related to the initial contact (M2), load acceptance (M3), and propulsion (M4).
\end{abstract}

\footnotetext{
${ }^{*}$ Corresponding author. Address: Department of Neurorehabilitation Engineering, Bernstein Focus Neurotechnology Göttingen, Bernstein Center for Computational Neuroscience, University Medical Center Göttingen, Georg-August University, Von-Siebold-Str. 4, 37075 Göttingen, Germany. Tel: + 49-(0)-551-3920100; fax: +49-(0)-5513920110.

E-mail address: dario.farina@bccn.uni-goettingen.de (D. Farina). Abbreviations: ADD, adductor muscles; BF, biceps femoris; CoM, centre of mass; CoMp, CoM power; CoV, coefficients of variations; EMG, electromyography; EOB, external oblique; ESP, erector spinae; $F x$, medial-lateral ground reaction force; $F y$, anterior-posterior ground reaction force; Fz, vertical ground reaction force; GMA, gluteus maximus; GME, gluteus medius; M1, motor module 1; M2, motor module 2; M3, motor module 3; M4, motor module 4; M5, motor module 5; PER, peroneus longus; RAB, rectus abdominis; RF, rectus femoris; SSE, sum of squared errors; SST, total sum of squares; ST, semitendinosus; TA, tibialis anterior; TFL, tensor fascia latae; VAF, variation accounted for; VL, vastus lateralis; VM, vastus medialis; W-Abs, external work during absorption; W-Prp, external work during propulsion.
}

The variability in timing between impulses across subjects was greater for cutting manoeuvres than for running. The timing difference between M2 and M3 in the cutting manoeuvres was significantly associated to W-Abs $\left(r^{2}=\right.$ 0.45 ) whereas the timing between $M 3$ and M4 was associated to $W$-Prp $\left(r^{2}=0.43\right)$. These results suggest that complex locomotor tasks can be achieved by impulsive activation of muscle groups, and that performance is associated to the specific timing of the activation impulses. (C) 2012 IBRO. Published by Elsevier Ltd. All rights reserved.

Key words: side-step cutting, motor modules, EMG, motor performance.

\section{INTRODUCTION}

It has been suggested that the many mechanical degrees of freedom to be controlled during locomotion are modulated by the CNS at a low-dimensional level, by activating sets of relative intensities or weightings (motor modules) of muscle activation that are recruited in a specific sequence. Such motor modules are believed to represent neural structures in the spinal cord, activated by descending neurons and central pattern generators, combined to afferent input to produce a wide range of movements (d'Avella et al., 2003; Ivanenko et al., 2005; Muceli et al., 2010; Lacquaniti et al., 2012b). A consistent observation in human locomotion is that four to six motor modules are activated by sequential impulses of activity that provide the timing for synchronous activation of muscles over time (Ivanenko et al., 2004, 2006; Cappellini et al., 2006; Gizzi et al., 2011; Lacquaniti et al., 2012b). The specific timing of activity of motor modules allows for a precise association to gait events, such as the initial contact, load acceptance, and push off (Ivanenko et al., 2008).

Although the muscle weightings are flexible and may change across tasks (Lacquaniti et al., 2012b) the timing of sequential impulsive control of locomotion is consistent across tasks and subjects (d'Avella et al., 2003; Ivanenko et al., 2004, 2006; d'Avella and Bizzi, 2005; Cappellini et al., 2006). For example, walking and running show similarly patterned control of neural commands (Cappellini et al., 2006; Lacquaniti et al., $2012 b)$ and become automatized motor gestures by experience, with primitive basic motor patterns which are innate (Lacquaniti et al., 2012a). When other tasks are added to a locomotion motor pattern, such as kicking during walking, additional modules and timing 
activation signals are added in a linear manner maintaining the basic structure of impulsive control (Ivanenko et al., 2005).

In this study, we analyse the neuromuscular organization of more complex locomotor tasks than those previously analysed. In these tasks, the subjects are requested to suddenly change the direction of running by $90^{\circ}$. These tasks, also called cutting manoeuvres, are characterized by a change in the original direction of straight running, which may require changes in the motor patterns to perform negative work, discontinuing the forward displacement, followed by the generation of an additional laterally directed impulse against the ground (Rand and Ohtsuki, 2000).

We hypothesized that such complex tasks are still controlled by the same burst-like impulses of activations as in walking and running with their respective timing being related to the main events during the change in direction. Verification of this hypothesis would show that the sequential impulsive control observed during walking/running is a general control strategy for locomotion, valid also for more complex locomotor tasks. Given the need for precise timing in complex tasks, it was further hypothesized that cutting manoeuvres, which are less common tasks than walking and running, would show a greater timing variability in activation impulses across subjects and that this variability would explain the differences in task performance across subjects. Verification of this hypothesis would associate a precise functional meaning to the timing of the neural commands to activate muscle groups in order to accelerate or decelerate the body's centre of mass (CoM).

The aim of the study was to verify the two hypotheses of sequential burst activation of a low number of motor modules in the complex task analysed and association of the activation timing to the biomechanical goals during the motion. We provide this analysis for a large number of subjects to identify subject-specific differences in the performance of the analysed task and association of these differences with the timing of neural control.

\section{EXPERIMENTAL PROCEDURES}

\section{Subjects}

Twenty-two healthy men (age, $28 \pm 4$ years; body mass, $71 \pm 10 \mathrm{~kg}$; body height, $171 \pm 7 \mathrm{~cm}$ ) volunteered for the experiment. All subjects were recreational practitioners of team sports (soccer, basketball, handball, ice hockey). They had no known history of neurological or motor disorder. All subjects provided written-informed consent before participation and the procedures were approved by the Ethical Committee of Northern Jutland (N-20100042).

\section{Experimental setup}

The subjects were asked to perform repeated $90^{\circ}$ cutting movements during a single session. The task consisted of running from 6 to $7 \mathrm{~m}$ away of a force platform, aiming to step with the right foot over the force platform, turn $90^{\circ}$ to the left and continue running (Fig. 1). All subjects were right-footed, which was defined by dominance test (drop and kick a ball against a wall three times), therefore all analyses were performed considering the dominant limb. Each subject performed $10-15$ cutting trials for familiarization being instructed to accelerate in a straight path towards the force platform and turn as fast as possible to the left. Adjustments on approaching running speed were necessary in order to ensure that subjects were performing the correct cutting trials as fast as possible. Subsequently, 10 cutting movements were recorded with a 40-60 s rest interval between trials to reduce the effects of fatigue. All subjects wore the same type of court shoes (FZ 2600W, FORZA ${ }^{\circledR}$, Brønderslev, Denmark) in order to keep consistent conditions for all subjects. Additionally, 10 of the subjects were asked to perform jogging at a self-selected (comfortable) speed on an $8 \mathrm{~m}$ walkway. Subjects performed 10 trials with a $30-\mathrm{s}$ rest interval between each trial.

\section{Data collection}

Kinematics. Retroreflective spherical markers were placed bilaterally each side of the subject to the skin overlying the following landmarks: calcaneus, first and fifth metatarsophalangeal joint, lateral malleolus, lateral condyle; greater trochanter, anterior superior iliac spine, posterior superior iliac spine and acromion. In addition, one marker was placed in the seventh cervical vertebrae, upper and lower endpoint (suprasternal notch and xiphoid process of the sternum). Further markers were placed bilaterally on lower extremity segments: one on each thigh, four on the leg and one on each arm, serving as tracking markers to define the 3D motion. The positions of the markers were tracked with a motion analysis system with eight infrared digital video cameras (Oqus 300 series, Qualisys, Gothenburg, Sweden). Kinematic data were recorded with a sampling frequency of $256 \mathrm{~Hz}$ and synchronized with the electromyography (EMG) and kinetic recordings. Subjects wore full stretch pants covering the EMG cables to avoid movement artefacts.

Kinetics. The vertical $(F z)$, anterior-posterior $(F y)$ and medial-lateral $(F x)$ ground reaction forces were recorded at $1024 \mathrm{~Hz}$ by a three-dimensional force platform (AMTI, OR6-5, Watertown, MA) constructed over a hydraulic system (van Doornik and Sinkjaer, 2007). Software developed in Labview platform (MrKick II, Aalborg University, Aalborg, Denmark) was used for recording. Using a feedback electric circuit, the $F z$ force also served as trigger signal to initiate the force plate movement.

EMG. Surface EMG signals were recorded in bipolar derivations with pairs of $\mathrm{Ag} / \mathrm{AgCl}$ electrodes (Ambu Neuroline 720 01-K/12; Ambu, Ballerup, Denmark) with $22 \mathrm{~mm}$ of centreto-centre spacing. Prior to electrode placement the skin was shaved and lightly abraded. The EMG signals were amplified with a gain of 2000 (EMG-USB, LISiN; OT Bioelettronica, Turin, Italy), sampled at $2048 \mathrm{~Hz}$, band-pass filtered (second-order, zero lag Butterworth, bandwidth $10-500 \mathrm{~Hz}$ ) and 12 bits per sample $A / D$ converted. A reference electrode was placed on the right wrist. The EMG signals were recorded from the following muscles of the right side according to the SENIAM recommendations (Hermens et al., 2000) and previous literature (Ivanenko et al., 2006): tibialis anterior (TA), peroneus longus (PER), soleus (SOL), gastrocnemius medialis (GM), vastus medialis $(V M)$, vastus lateralis $(V L)$, rectus femoris (RF), biceps femoris (BF), semitendinosus (ST), adductor muscles (ADD), gluteus medius (GME), gluteus maximus (GMA), tensor fascia latae (TFL), erector spinae at L1 (ESP), rectus abdominis (RAB) and external oblique (EOB). 


\section{A ó 3D model}

cuttng manoeuvre

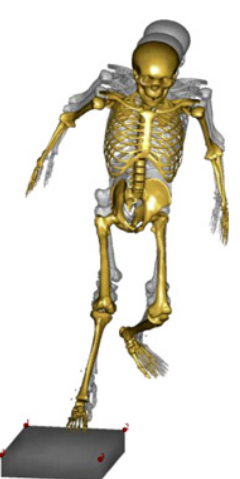

1 ó landing preparation

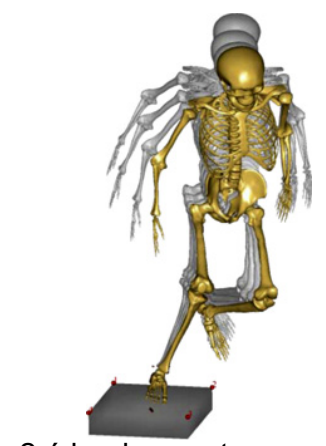

2 ó load acceptance

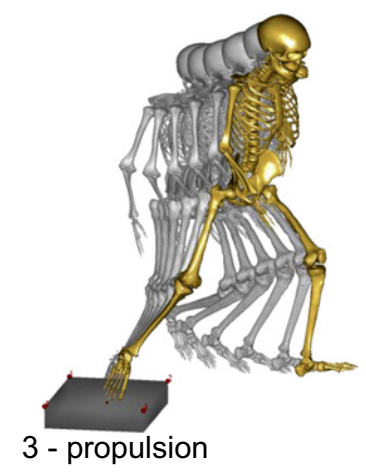

B ó kinematics and kinetics

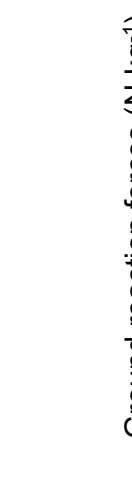

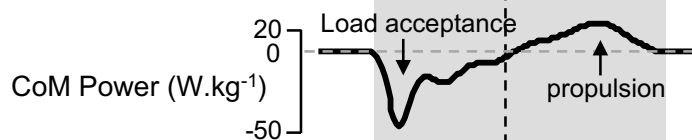
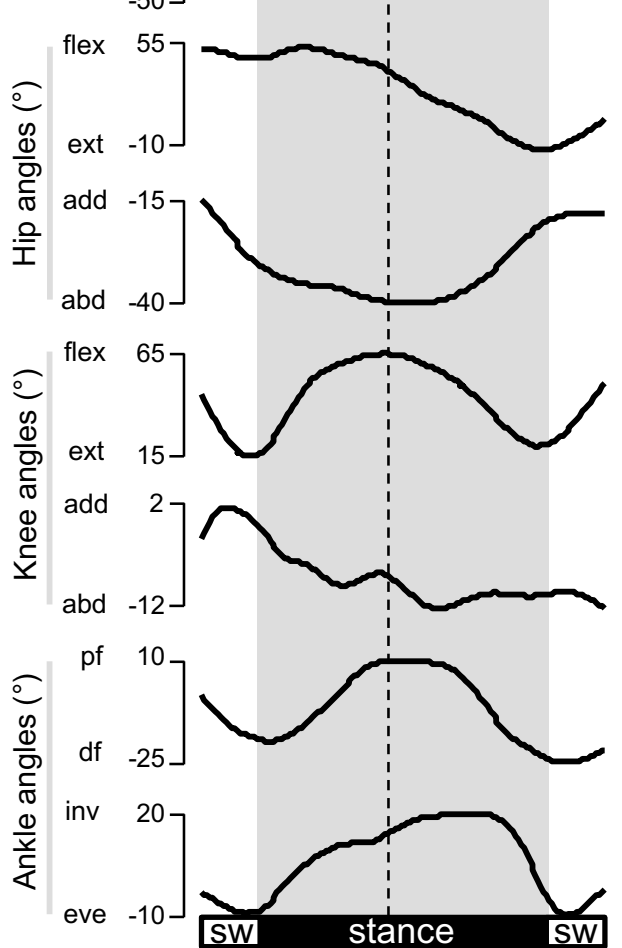

Fig. 1. Representative data from one subject performing a cutting movement. (A) 3D model describing the approach to initial contact (A1), load acceptance (A2) and propulsion (A3). On the right (B) ground reaction forces ( $F x, F y$ and $F z$ ), centre of mass (CoM), power and joint angles for the hip knee and ankle are illustrated in a period before, during (grey area) and after the stance phase. The dashed vertical line indicates the transition from the absorption period to the propulsion period of the cutting.

\section{Data analysis}

For the kinematic analysis, the body was modeled as an interconnected chain of rigid segments: foot, shank, thigh, pelvis, trunk and arms. Segment lengths were scaled to match motion capture data over the entire trial, using the model and methods described by Andersen et al. (2010). Inverse kinematic analysis was applied to calculate the trunk CoM, joint angles and angular velocities between segments using the AnyBody Modelling System 5.1 (Anybody Technology, Aalborg, Denmark), a multibody modelling system for musculoskeletal biomechanics. The segmentation for EMG factorization was defined from the left initial contact prior the right foot step on the force platform to the end of the stance phase on the force platform. The left initial contact was defined from the foot kinematic data, whereas the end of the stance phase for the right leg was determined by the force plate recordings (when the vertical ground reaction force fell below $20 \mathrm{~N}$ ). The body CoM, CoM power (CoMp: sum of forces $*$ CoM velocity [watts/ body weight]), and joint angles were calculated during the period of contact to the force platform. The external work (Saibene and Minetti, 2003) was calculated by the integration of CoMp during the absorption period (W-Abs, defined as the integral of the negative power) and during the early propulsion period (W-Prp, defined from the zero crossing of CoMp to end of ground contact. CoMp, see Fig. 1 for illustration). The external work has been used as a reliable estimation of the work done to raise and accelerate the CoM during locomotion 
tasks (Saibene and Minetti, 2003). The CoM acceleration directly reflects the body displacement generated by the forces applied to the ground (Cavagna, 1975; Saibene and Minetti, 2003; Hamner et al., 2010), therefore enhanced performance requires more work to be generated/less work to be absorbed within a fixed time period. This concept was used in the present investigation to justify the use of external work to describe performance during cutting manoeuvres.

Signal processing. After segmentation, the surface EMG signals from the 16 muscles were full-wave rectified, low-pass filtered $(10 \mathrm{~Hz})$ and time-normalized in order to obtain 200 data points for one gait cycle (d'Avella et al., 2003; Ivanenko et al., 2004). For each subject, all trials for a given condition were averaged, followed by the application of non-negative matrix factorization (NMF) (d'Avella et al., 2003; Gizzi et al., 2011) for identification of motor modules and activation signals (Fig. 2).

Motor module model. The EMG signals recorded from $M$ muscles were indicated as

$X(k)=\left[x_{1}(k), x_{2}(k), \ldots, x_{M}(k)\right]^{T}$

where $x_{M}(k)$ is the activity of the $m$ th muscle at the time instant $k$. The activation signals $P(k)$ were indicated as $(N<M)$ :

$P(k)=\left[p_{1}(k), p_{2}(k), \ldots, p_{N}(k)\right]^{T}$

The relation between $X(k)$ and $P(k)$ is described as follows:

$X(k) \approx X_{r}(k)=S \cdot P(k)$

where $X_{r}(k)$ is the muscle activity vector reconstructed by the factorization. In Eq. (3), the EMG $X(k)$ are obtained by linear transformation of the activation signals $P(k)$ with gain factors $S_{m n}$. The matrix whose columns were the weights of each activation signal for each muscle is denoted as $S$ in Eq. (3) and will be referred to as the motor module matrix (Lee and Seung, 1999).

Dimensionality. The number of motor modules $N$ needed for accurate description of the movement was assessed by the dimensionality analysis proposed by (d'Avella et al., 2003). According to this procedure, the quality of reconstruction of the muscle activation pattern is analysed as a function of the number of modules and the minimum number of modules is identified as the point in which this curve pronouncedly changes its slope (d'Avella et al., 2003). In addition to this criterion, a minimum threshold for reconstruction quality was set at $80 \%$. For quantifying the quality of reconstruction, the estimated muscular activation pattern was compared with the recorded pattern by means of the variation accounted for (VAF) value defined as the variation that can be explained by the model: $\mathrm{VAF}=1-\mathrm{SSE} / \mathrm{SST}$, where SSE (sum of squared errors) is the unexplained variation and SST (total sum of squares) is the pooled variation of the data.

After computation of the reconstruction quality, the motor modules for each subject were extracted from the concatenation of all trials. Similarities among the different subjects were investigated for motor modules and activation signals. The motor module matrices were compared by computing the scalar product between pairs of columns, normalized by the product of the norms of each column (d'Avella et al., 2003; Torres-Oviedo and Ting, 2007; Muceli et al., 2010). Similarities between activation signals were quantified by the value of the cross-correlation function at zero time lag (Clark et al., 2010; Gizzi et al., 2011). In addition, the EMG activities from all subjects were concatenated for a given condition, from which motor modules were extracted to represent the whole group of subjects. In this manner, all the

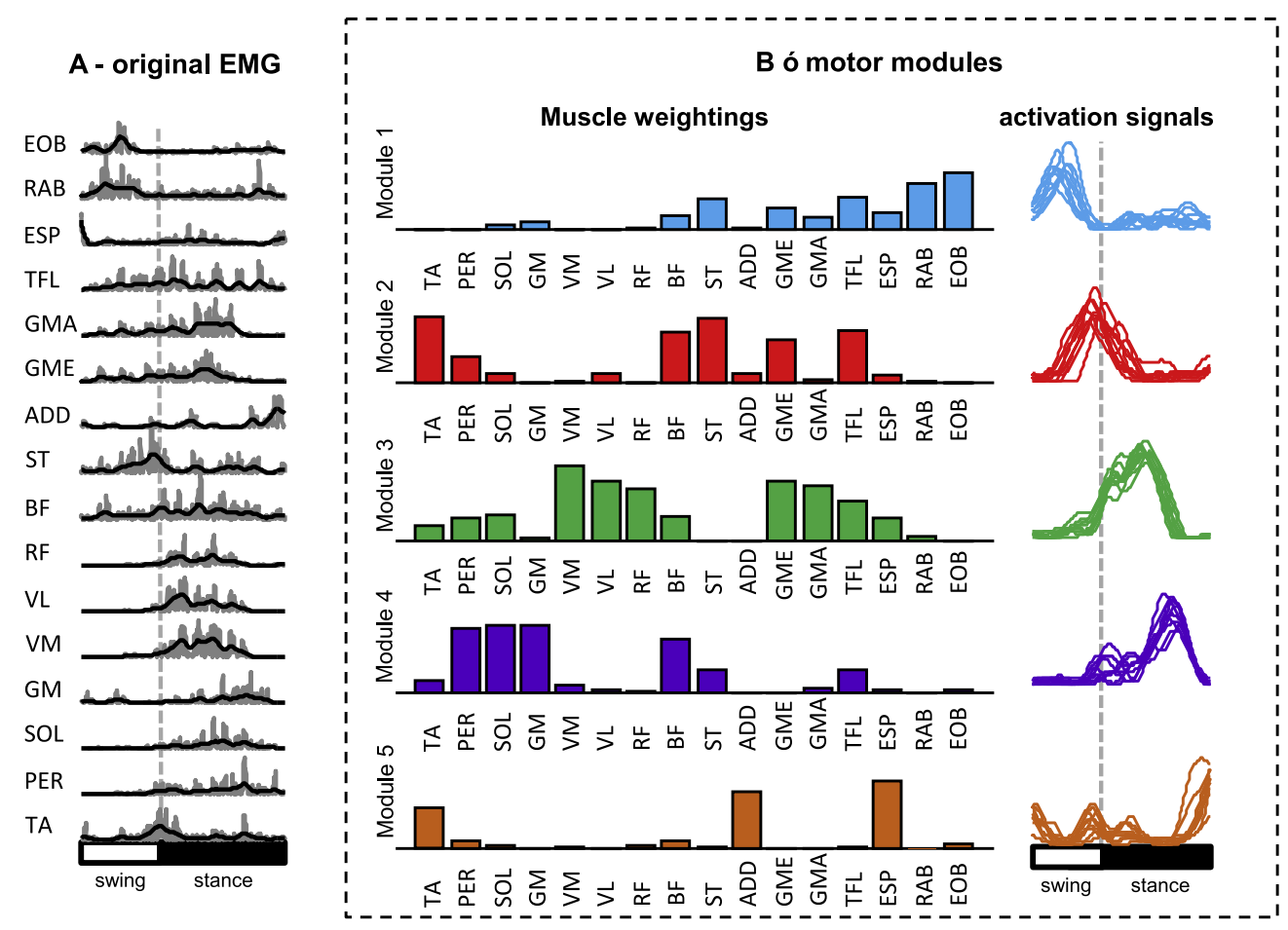

\section{C - reconstructed signals}

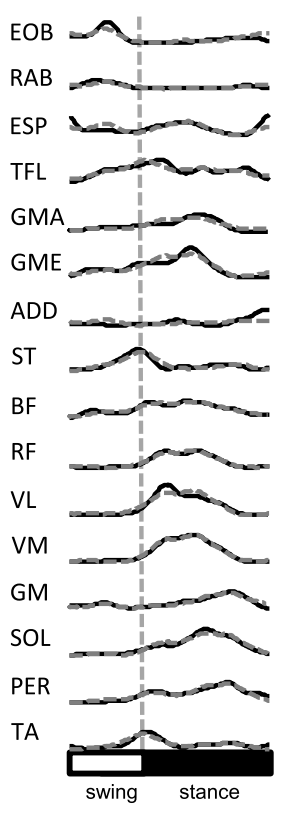

Fig. 2. Reconstruction of EMG signals by non-negative matrix factorization (NMF) for a representative subject in 10 cutting manoeuvres. (A) Raw rectified muscle activation (grey lines) and low-pass filtered envelopes (black lines), throughout one cutting cycle. (B) Muscle activity was processed by a NMF algorithm, which reconstructs the original EMG using a small set of modules. (C) Original (black solid lines) and reconstructed EMG from the multiplication of muscle weightings and activation signals (grey dashed lines on top of black lines). 
variability in the dataset was taken into account. The timing of each activation signal was defined as the time instant of maximum value of the activation signal as a percentage of the running/cutting cycle.

Kinematic data were low-pass filtered $(10 \mathrm{~Hz}$, second-order, zero lag Butterworth) CoM mass speed was computed from 200 to $100 \mathrm{~ms}$ before right foot contact to the force platform. Joint angles/angular velocities were analysed qualitatively from the averaged data across all trials and subsequently averaged across subjects. For each activation signal respective to the motor modules from running and cutting, the peak timing of the curves ( $\%$ of cutting cycle) was defined as the maximum value of the bursts. The bursts were automatically selected by specific algorithms and manually checked by the researchers for consistency. Coefficients of variations (CoV) were calculated for the kinematics and ground reaction force variables as well as for the timing of the activation signals from running and cutting. The Pearson coefficient of determination, calculated from specific statistical software package (SPSS 19, Chicago, IL), was used to explain the relation between (1) the external work generated during the absorption period (W-Abs) in relation to the timing of $\mathrm{M} 3$ (propulsion) and the external work generated during the propulsion period (W-Prp) in relation to the net change in time from M2 to M3 ( $\Delta$ Prop); (2) the external work absorbed during the initial phase of the propulsion in relation to the timing of M4 (push-off) and to the net change in time from M3 to M4 ( $\Delta$ Push-off); and (3) the total external work generated/absorbed during stance phase in relation to the net change in time from $M 2$ to $M 4$ ( $\Delta$ Stance). The significance level for the linear regressions was set to $p<0.05$.

\section{RESULTS}

The running task was performed at approximately $9 \mathrm{~km} \mathrm{~h}^{-1}\left(\min 8.67 \mathrm{~km} \mathrm{~h}^{-1}, \max 9.3 \mathrm{~km} \mathrm{~h}^{-1}\right)$. For the cutting task, the stance duration, peak ground reaction forces and trunk CoM speed $100 \mathrm{~ms}$ before initial contact had low variability among subjects (Table 1 ). The trunk CoM speed was on average $2.67 \mathrm{~m} \mathrm{~s}^{-1}$ $\left(9.6 \mathrm{~km} \mathrm{~h}^{-1}\right)$ and also showed low variability (CoV $<20 \%$ ). On the other hand, the distance of the left foot in the step prior to the cutting manoeuvre showed high variability among the subjects ( $\mathrm{CoV}>50 \%$; Table 1$)$.

\section{Kinematics of cutting movements}

The stance phase for the cutting manoeuvre started with hip flexed $\left(25 \pm 2.3^{\circ}\right)$, knee abducted $\left(20 \pm 1.1^{\circ}\right)$ near extension $\left(17 \pm 2.4^{\circ}\right)$, and ankle dorsiflexed $\left(-16 \pm 3.7^{\circ}\right)$ (Fig. 3). During the first half of the stance phase, the hip and knee flexion were combined with progressively increased external rotation and ankle plantar flexion. Subsequently, there was a general extension for the hip, knee and ankle in order to generate propulsion. The change in direction in the last $25 \%$ of the stance period involved hip adduction and external rotation in addition to ankle eversion towards the push-off event.

\section{Dimensionality}

The analysis of dimensionality from single trials determined that five motor modules were required to reconstruct unilateral muscular activation for both running (average $\mathrm{VAF}=0.93 \pm 0.02 \%$ ) and cutting manoeuvres (average $\mathrm{VAF}=0.91 \pm 0.02)$. On average, VAF reached $90 \%$ (range from 0.89 to 0.97 ) with five modules, and the addition of a sixth module only increased VAF by $2.0 \pm 0.6 \%$ (average over all subjects from the running and cutting tasks). The dimensionality from the concatenation of all trials for each subject also indicated that five modules were sufficient to reconstruct the muscular activation pattern of running with VAF $>90 \%$ (0.92 $\pm 0.03 ; 10$ subjects), but cutting movements showed lower quality for the concatenations (average $=$ $0.81 \pm 0.04 ; 22$ subjects).

\section{Motor modules in running and cutting manoeuvres}

Running motor modules (Fig. 4A) showed modular organization that resembled previously reported data (Cappellini et al., 2006). The first module was related to heel strike and load acceptance, consisting of TA, quadriceps and hip extensors. The second module was related to push-off that ends the stance phase, by the recruitment of plantar flexor muscles. The third module consisted of trunk muscles during the transition from stance to swing phase. The fourth module consisted of the activation of TA, RF, ADD, TFL and ESP, in order to perform swing. The fifth module had predominant activation of hamstrings and hip extensors, together with ESP and EOB, responsible for hip and trunk stabilization prior to landing.

Fig. 2 shows the motor modules obtained during cutting manoeuvres for a representative subject ordered by the timing of the activation signals. Module 1 (M1) consisted in the activation of hip and trunk muscles prior to the initial contact to the force platform. BF and ST decelerate the knee joint flexion and GME, TFL, RAB and EOB stabilize the hip and trunk prior to landing. Module 2 (M2) was related to the foot strike event, which consisted of the synchronous activation of foot stabilizers (TA and PER), hamstrings, and lateral hip stabilizers in the frontal plane (GME, TFL). The third module (M3) was related to the impact, absorption and propulsion, where the predominant muscles are the quadriceps muscles (VL, VM and RF) and hip extensors (GME, GMA). The fourth module (M4) was related to the push-off in the final phase of the contact to the force platform. This module

Table 1. Mean (SD) and coefficient of variation (CoV) of stance duration (stc_dur), vertical peak force during absorption phase (pk_abs), propulsion phase (pk_prp), lateral peak force to push-off (pk_lat), distance of left foot to the force platform previous to right initial contact (left_dist) and centre of mass speed $100 \mathrm{~ms}$ before right initial contact to the platform (CoM_speed) for all measured subjects $(n=22)$

\begin{tabular}{lllllc}
\hline & stc_dur $(\mathrm{ms})$ & pk_abs $\left(\mathrm{N} \mathrm{kg}^{-1}\right)$ & pk prp $\left(\mathrm{N} \mathrm{kg}^{-1}\right)$ & pk_lat $\left(\mathrm{N} \mathrm{kg}{ }^{-1}\right)$ & left_dist $\left(\mathrm{cm}^{-1}\right)$ \\
\hline Mean \pm SD & $327 \pm 50$ & $27.9 \pm 7$ & $18 \pm 2.4$ & $7.3 \pm 1.9$ & $55.7 \pm 30$ \\
CoV $(\%)$ & 15.49 & 26.32 & 13.41 & 25.63 & 54.39 \\
\hline
\end{tabular}




\section{Joint angles for cutting manoeuvres}
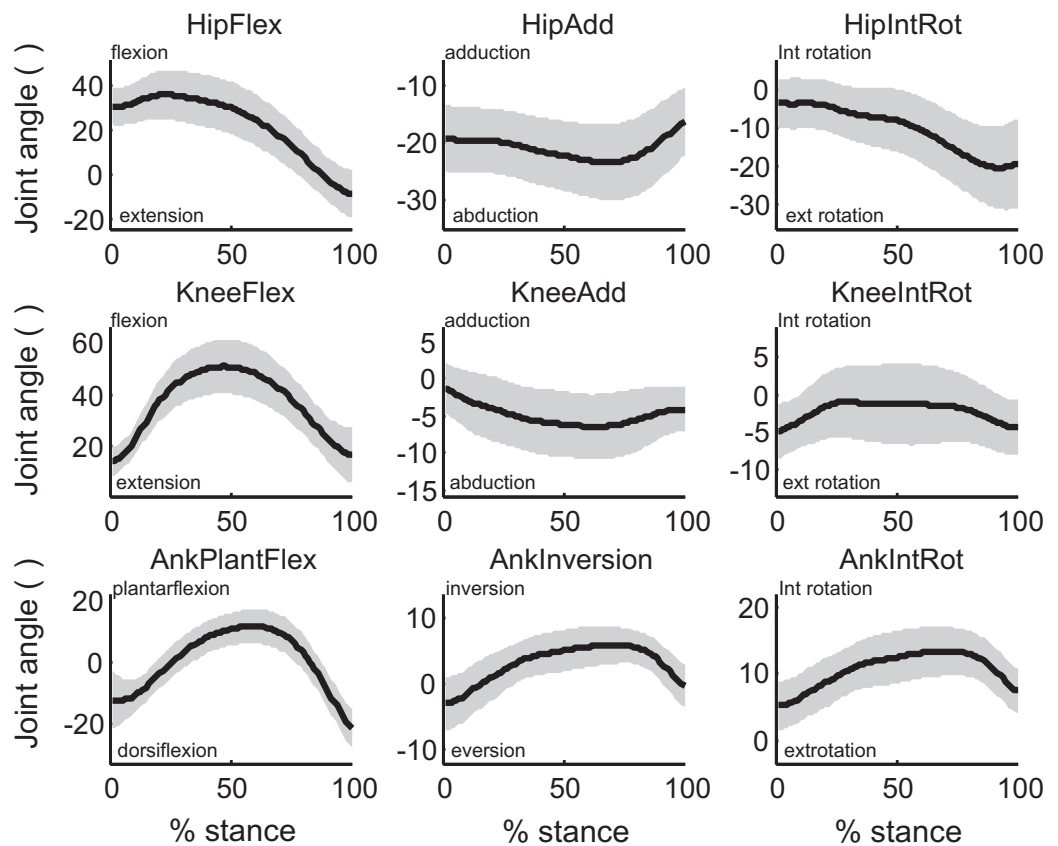

Fig. 3. Mean (black lines) and 1 SD range (grey area) of the right limb joint angles during the stance period of cutting manoeuvres for all subjects $(n=22)$.

A - RUNNING Muscle weightings

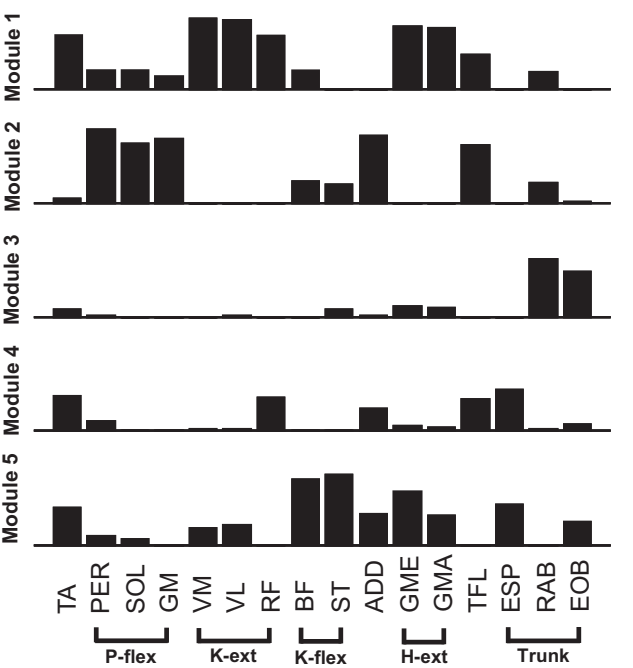

activation signals

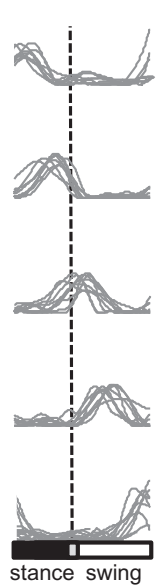

$\mathrm{s}=0.95$ with $\mathrm{M} 3$

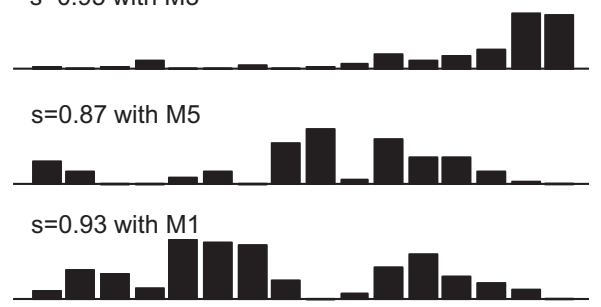

$\mathrm{s}=0.86$ with $\mathrm{M} 2$

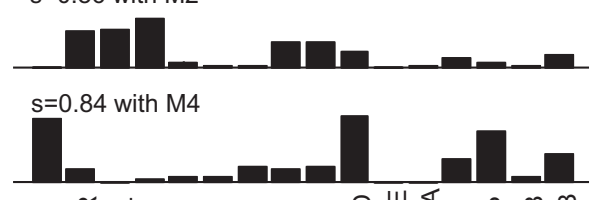

『㟧 ウ

B - CUTTING

Muscle weightings

activation signals

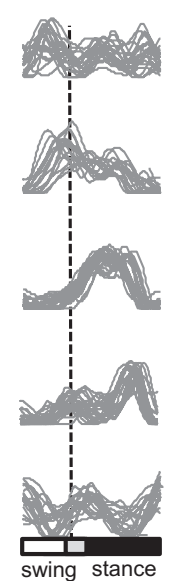

$$
\text { P-flex } \underset{\text { K-ext }}{\mathbf{K} \text { K-flex }} \underset{\text { H-ext }}{\mathbf{L}} \text { Trunk }
$$

Fig. 4. Motor modules that represent running (A, $n=10)$ and cutting $(\mathrm{B}, n=22)$. "s" on top of weighting coefficients for cutting correspond to similarities with respect to weighting coefficients from running. Abbreviations of the muscle nomenclature are described in the Methods (Section Data collection). P-flex, plantar flexors; K-ext, knee extensors; K-flex, knee flexors; H-ext, hip extensors.

consisted of the activation of plantar flexors and hamstrings. The fifth module (M5) consisted of the activation of TA, ADD and ESP, during swing, initial contact and predominantly at the end of stance. This module may be responsible for modulating ankle and hip stability while turning to the left as well as for maintaining the trunk erect posture.

\section{Comparison between running and cutting tasks}

For both running (Fig. 4A) and cutting tasks (Fig. 4B), temporally ordered impulses of activity modulated the weightings of the muscle set. Running was segmented from initial contact to initial contact, whereas cutting movements were segmented from maximal right knee 
flexion to right toe-off from the platform. The concatenation of all subjects for cutting manoeuvres also revealed similar functionality for the motor modules shown in Fig. 2 as well as a similar timing pattern in relation to the cycle (swing or stance phase) for its activation.

The similarity analysis revealed that weighting coefficients from running were similar to those of cutting movements (similarities $>0.80$; Fig. 4B). Moreover, it was possible to qualitatively identify similarities for the timing of the peaks with respect to the cutting phase (stance or swing). For instance, both M2 from running task and M4 from cutting task showed peak activation timing towards the end of the stance phase. Although high similarity was found there were also differences in motor modules between the two tasks. For instance, weighting for $\mathrm{M} 2$ during cutting did not include trunk muscles (ESP, EOB), which were recruited in M5 instead in order to assist the trunk rotation to the left. These results suggest that running and cutting tasks show similar modular organization, which has specific timing modulation to recruit the necessary muscles to accomplish the motor gesture. However, the apparently complex change in direction is accomplished by slight changes in few muscle weightings, together with temporal adjustments of the activation signals.

\section{Intra- and inter-subject similarity}

Similarity was computed for each individual separately by comparing muscle weightings and activation signals from one cutting manoeuvre to all the others (Table 2). The

Table 2. Mean (SD) and range of inter-trials similarity for weighting coefficients (motor modules) of each individual during cutting manoeuvres

\begin{tabular}{lll}
\hline & \multicolumn{2}{l}{ Similarity - motor modules } \\
\cline { 2 - 3 } & Mean $\pm \mathrm{SD}$ & Range \\
\hline Sub-1 & $0.84 \pm 0.06$ & $0.75-0.92$ \\
Sub-2 & $0.83 \pm 0.06$ & $0.65-0.95$ \\
Sub-3 & $0.86 \pm 0.06$ & $0.77-0.94$ \\
Sub-4 & $0.85 \pm 0.06$ & $0.73-0.90$ \\
Sub-5 & $0.86 \pm 0.05$ & $0.75-0.94$ \\
Sub-6 & $0.81 \pm 0.07$ & $0.72-0.91$ \\
Sub-7 & $0.86 \pm 0.05$ & $0.77-0.95$ \\
Sub-8 & $0.82 \pm 0.06$ & $0.74-0.94$ \\
Sub-9 & $0.84 \pm 0.05$ & $0.77-0.94$ \\
Sub-10 & $0.86 \pm 0.05$ & $0.78-0.96$ \\
Sub-11 & $0.86 \pm 0.05$ & $0.75-0.95$ \\
Sub-12 & $0.81 \pm 0.07$ & $0.71-0.92$ \\
Sub-13 & $0.79 \pm 0.07$ & $0.71-0.88$ \\
Sub-14 & $0.82 \pm 0.07$ & $0.70-0.94$ \\
Sub-15 & $0.84 \pm 0.07$ & $0.76-0.96$ \\
Sub-16 & $0.87 \pm 0.06$ & $0.77-0.96$ \\
Sub-17 & $0.87 \pm 0.04$ & $0.80-0.95$ \\
Sub-18 & $0.91 \pm 0.04$ & $0.84-0.97$ \\
Sub-19 & $0.83 \pm 0.07$ & $0.76-0.88$ \\
Sub-20 & $0.82 \pm 0.06$ & $0.73-0.93$ \\
Sub-21 & $0.83 \pm 0.06$ & $0.76-0.91$ \\
Sub-22 & $0.86 \pm 0.05$ & $0.81-0.91$ \\
Total & $0.84 \pm 0.03$ & $0.75-0.93$ \\
\hline & &
\end{tabular}

Table 3. Mean (SD) and range of similarities among subjects for the muscle weightings and activation signals of the five motor modules extracted from the cutting manoeuvres

\begin{tabular}{llllll}
\hline & \multicolumn{2}{l}{ Muscle weightings } & & \multicolumn{2}{l}{ Activation signals } \\
\cline { 2 - 3 } \cline { 5 - 6 } & Mean \pm SD & Range & & Mean \pm SD & Range \\
\hline M1 & $0.74 \pm 0.11$ & $0.52-0.96$ & & $0.51 \pm 0.11$ & $0.32-0.96$ \\
M2 & $0.71 \pm 0.09$ & $0.43-0.94$ & & $0.71 \pm 0.12$ & $0.50-0.96$ \\
M3 & $0.82 \pm 0.07$ & $0.51-0.98$ & & $0.77 \pm 0.12$ & $0.45-0.99$ \\
M4 & $0.76 \pm 0.10$ & $0.51-0.95$ & & $0.76 \pm 0.13$ & $0.47-0.98$ \\
M5 & $0.72 \pm 0.10$ & $0.50-0.94$ & & $0.52 \pm 0.10$ & $0.24-0.88$ \\
\hline
\end{tabular}

Table 4. Mean (SD) peak activation timing (\% of the cutting cycle) for the five motor modules that describe running and cutting manoeuvres. Coefficient of variation (CoV) was also calculated (standard deviation/ mean)

\begin{tabular}{lclllll}
\hline & \multicolumn{2}{l}{ Running } & & \multicolumn{2}{l}{ Cutting } \\
\cline { 2 - 3 } \cline { 5 - 6 } & Mean \pm SD & CoV $(\%)$ & & Mean \pm SD & CoV $(\%)$ \\
\hline M1 & $8.2 \pm 0.6$ & 8.1 & & $15.4 \pm 4.6$ & 29.8 \\
M2 & $34.8 \pm 1.9$ & 5.7 & & $37.2 \pm 6.1$ & 16.4 \\
M3 & $49.7 \pm 3.5$ & 7.0 & & $63.7 \pm 10.6$ & 16.8 \\
M4 & $75.7 \pm 1.94$ & 2.6 & & $82.3 \pm 9.4$ & 11.4 \\
M5 & $92.4 \pm 3.3$ & 3.6 & & $81.4 \pm 23.4$ & 28.6 \\
\hline
\end{tabular}

muscle weighting coefficients showed a mean inter-trial similarity of 0.84 and low inter-subject variability. Motor modules extracted from cutting manoeuvres of different subjects showed an average similarity of 0.75 . When the different motor modules were analysed separately, the highest values for similarities were found for M2, M3 and M4 (Table 3).

\section{Activation signals}

The variability in activation signal timing, as measured by CoV, was smaller for running (range $\sim 3-8 \%$; Table 4 ), than cutting manoeuvres (especially for M1 and M5, with $\mathrm{CoV} \sim 28 \%$ ). The motor modules related to forward/ lateral propulsion by the plantar flexors showed the lowest CoV for both running (M2) and cutting tasks (M4).

The difference in variability of timing between the two tasks is evident in Fig. 5, which shows the five motor modules and their respective activation signals for running (Fig. 5A and B) and cutting manoeuvres (Fig. 5C and D). Colour maps that represent the activation signals from running show robust inter-subject peak timing position (vertical dashed traces), with low variation in time (the grey area around the traces represents $\pm S D$ ). The timing for cutting manoeuvres had greater variability, especially for M1 and M5.

\section{Activation timing and task performance}

There were significant associations between $\mathrm{W}$-Abs and $\Delta$ Prop $\left(r^{2}=0.45,95 \%\right.$ confidence interval $=-26.2$ to $-18,1, p<0.001$; Fig. 6A), between W-Prp and $\Delta$ Pushoff $\left(r^{2}=0.43, p<0.001\right.$; Fig. 6B), and between the total external work during the stance phase and $\Delta$ Stance $\left(r^{2}=0.35, p<0.001\right.$; Fig. $\left.6 C\right)$. 

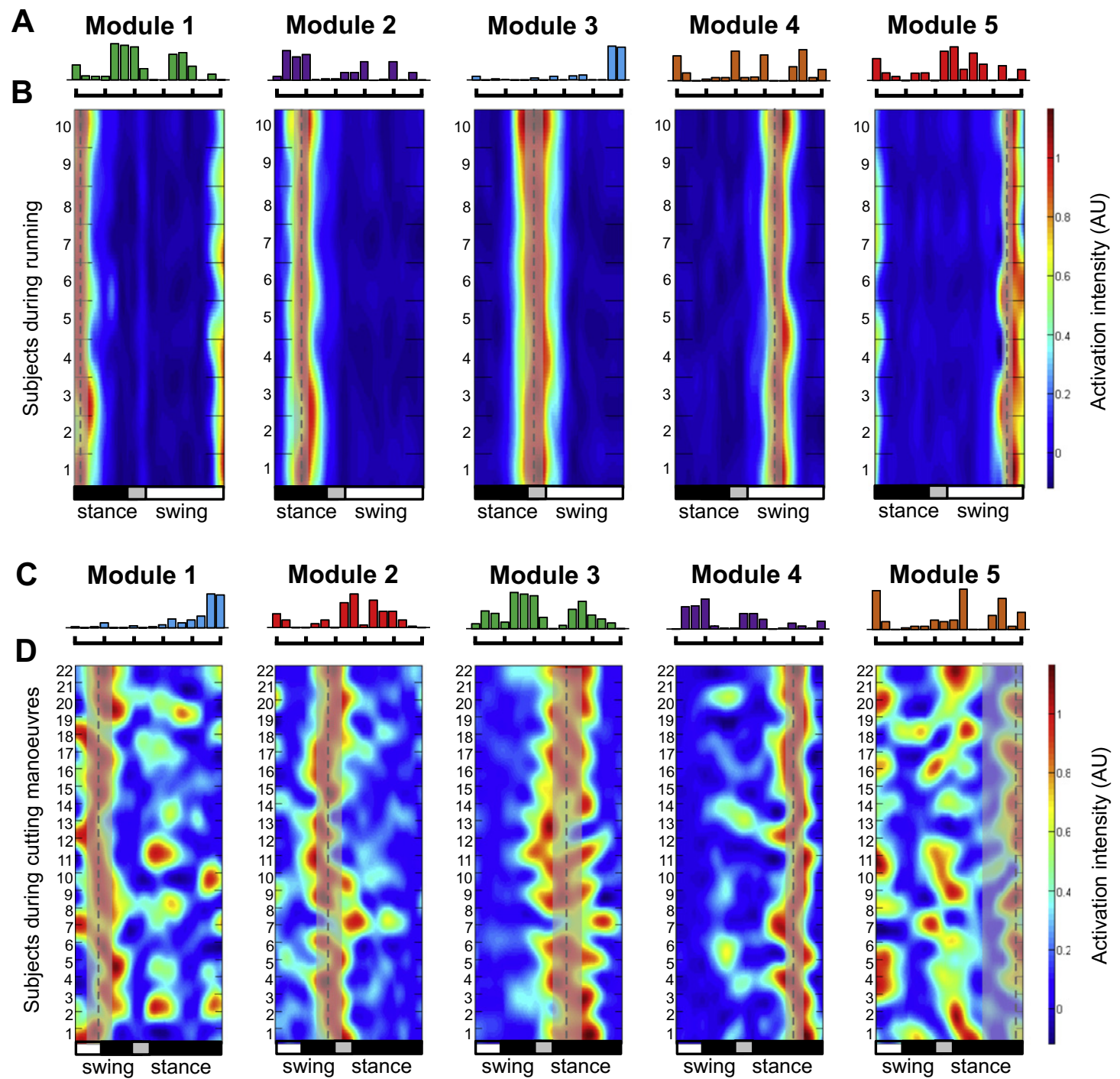

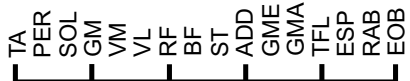

Fig. 5. Motor modules that describe running and cutting manoeuvres. At the top, running weighting coefficients (A) and activation signals from all subjects represented in a colour map (A). In the same way, at the bottom, weighting coefficients $(C)$ and activation signals from all subjects represented in a colour map (D) for cutting manoeuvres. Homologous motor modules in running and cutting manoeuvres are represented by the same colour bars. The vertical traces indicate the position of mean timing for the main peak throughout the cycles. The grey area surrounding the mean values represent the standard deviation for the peak timing. The running and cutting cycles are represented at the bottom of the colour bars. The grey area represents the standard deviation of the transition from stance to swing (running) or swing to stance (cutting). The order of the muscles in the motor modules is shown at the bottom of the figure.

\section{DISCUSSION}

The neural control of cutting manoeuvres is determined by a sequence of activation impulses that act on a small set of muscle weightings, in a similar fashion as during running. Although a similar inter-subject kinematics for the cutting task, the specificity of the motor action and consequent complexity is noticeable by the higher variability in the timing of the activation signals. This higher variability in timing for cutting manoeuvres could be partly explained by inter-subject variability in absorbing external work during the stance phase. Changes in direction indeed require optimization of the external work conversion during the impact absorption and the following propulsion. Therefore, the amount of external work conversion was associated to the relative timing or switching between motor modules.

Previous literature has shown that running can be represented by the same motor modules found during walking, with differences in timing of the activation signals to determine the biomechanical goals throughout the gait cycle (Cappellini et al., 2006; Ivanenko et al., 2006). Moreover, cyclic movements, such as walking, running and swimming, may have the same modular organization (d'Avella et al., 2003). Therefore, there is strong evidence suggesting that muscular coordination 

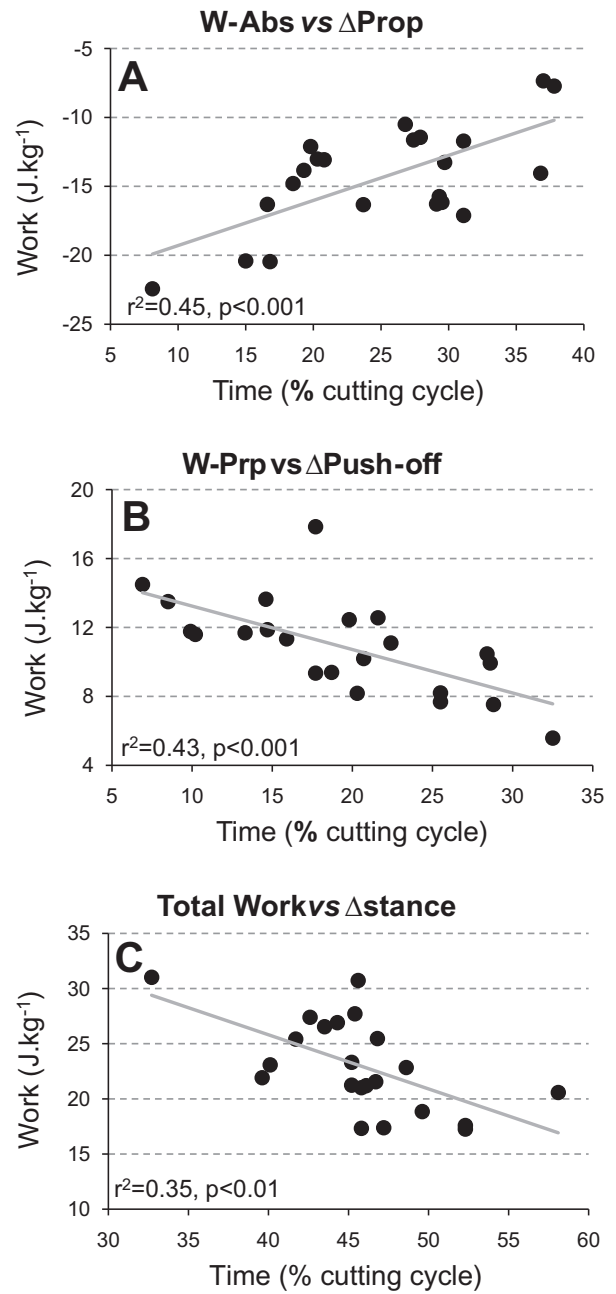

Fig. 6. Coefficient of determination $\left(r^{2}\right)$ and significance level $(p)$ for the relationship between $(A)$ external work for the absorption phase (W-Abs) and the time window between the peaks of activation signals of M2 to M3 ( $\triangle$ Prop, expressed as \% of the cutting cycle) and (B) external work for the propulsion phase (W-Prp) and the time window between the peaks of activation signals of M3 to M4 ( $\triangle$ Push-off, expressed as \% of the cutting cycle). (C) Total external work generated during stance phase and the time window between peaks of activation signals of $\mathrm{M} 2$ to $\mathrm{M} 4$.

for a given movement relies on intrinsic motor patterns that are activated in the most adequate timing. Our results complement this evidence by suggesting that discrete complex locomotor movements can also be described by a few motor modules that may be intrinsic to individuals with different previous exercise experience and anthropometric characteristics. The motor modules and activation signals that control running in the present investigation are comparable to previous literature (Cappellini et al., 2006; Ivanenko et al., 2008). Despite slight differences in the selection of muscles, the functionality of the described motor modules and especially the activation timing are in agreement with previously reported motor patterns for running (Cappellini et al., 2006).

The values for muscle weightings in cutting manoeuvres were similar to those of running. Cutting tasks involve essentially similar biomechanical goals as running, that accordingly require the activation of similar muscles. For instance, the recruitment of knee and hip extensors during load acceptance and propulsion, or the activation of plantar flexors and hamstrings (acting as hip extensors) to perform push-off were found in the present study and also in previous investigations concerning human walking and running (Ivanenko et al., 2004, 2005; Cappellini et al., 2006; Clark et al., 2010; Gizzi et al., 2011). These similarities suggest that the locomotor events in cutting tasks and running are explained by similar motor modules, even though cutting manoeuvres are non-cyclic and require a fast change in direction. Despite predominant similarities between modules from running and cutting manoeuvres, there were specific changes that determined the differences between the two tasks. The motor module related to landing during cutting manoeuvres (M2) showed reduced weighting for ADD and increased weighting for TFL when compared to the homologous module during running (M5). These slight changes are essential to allow the optimal motor performance during the task and reinforce previous concepts that the flexibility of motor modules allows for the performance of many different motor behaviors (Ivanenko et al., 2004; d'Avella and Bizzi, 2005; Lacquaniti et al., 2012b). It is also important to highlight that the change in direction during cutting manoeuvres may also be related to the recruitment of contralateral muscles especially in the trunk, which were not included in the present analysis. Trunk orientation is essential to assure a safe performance during cutting manoeuvres, since increased torso leaning in the opposite direction of the cutting increase peak valgus and internal rotation moments on the knee joint (Dempsey et al., 2007). In addition, the positioning of the trunk influences the generation of braking forces that are important to prevent over-rotations of the body with respect to the CoM alignment (Jindrich et al., 2006). Therefore, a correct balance between the recruitment of bilateral trunk muscles seems necessary in order to stabilize the upper body. Future studies should be dedicated to understand the modulation of bilateral trunk muscles during cutting manoeuvres.

Previous investigations have described muscular coordination for cutting manoeuvres (Neptune et al., 1999; Rand and Ohtsuki, 2000; Besier et al., 2003) and most of the functional roles for each muscle/muscular group were confirmed in the present experiment by using EMG factorization instead of individual timing profiles. A more recent investigation has used computer-based modelling of running in order to describe individual muscle contributions to the CoM displacement (Hamner et al., 2010). The authors suggested that during the braking phase of stance there is predominant activation of the quadriceps muscles, whereas ankle plantar flexors are the main contributors to the propulsion phase. The present description of muscular patterns of activation for running and cutting manoeuvres corroborates the results from Hamner and co-workers (2010) from a neurophysiological perspective, by suggesting that neural control of such 
locomotor tasks might be partitioned in motor modules linked to functional goals such as landing, braking and propulsion.

An impulsive pattern for muscle recruitment was found for both running and cutting manoeuvres in the present investigation, which is in agreement with previous investigations of human locomotion (Ivanenko et al., 2005, 2006; Lacquaniti et al., 2012b). The activation impulses for the running task had smaller overlapping in time and lower variability in timing across subjects than those of cutting tasks. These differences may be explained by the fact that humans have a wellestablished motion pattern for walking and running (Lacquaniti et al., 2012). Cutting manoeuvres are tasks of greater complexity and less frequent with respect to walking/running and can be performed with different degrees of efficiency.

The present results suggest that differences in activation timing across individuals can alter performance, allowing individuals with faster transitions between motor modules to generate more external work. The absorption period of a cutting cycle is defined by a negative power generation that decelerates the CoM. The energy is mostly absorbed by eccentric knee and hip flexion, which initiate the subsequent propulsion. The propulsion period is characterized by a positive external work to perform the sideward displacement by knee and hip extension and subsequent push-off. The amount of external work generated during sports gestures varies with experience, since the motor gesture can be optimized to become faster and/or more precise (Sigward and Powers, 2006). Enhancements related to motor learning or practice are the facilitation of agonist and reduced antagonist activity, generating a smoother movement (Gabriel et al., 2006). The faster the transition from $M 2$ to $M 3$, the higher the En-ABS, which indicates that individuals more adapted to switch modules can optimize (i.e., increase) the impact absorption to perform a faster movement. In the same way, greater En-PRP indicates that subjects can perform the propulsion and push-off more effectively by generating greater muscular power, likely on the basis of a faster transition from M3 to M4.

Our results revealed that the ability to perform the subphases of cutting movements is strictly linked to an overall coordination that controls the movement. This coordination involves the activation of subsets of muscles in the adequate timing that slightly varies among individuals. Therefore, the sensory-motor integration of muscular actions and reaction forces has to be solved to perform the manoeuvre, and the transitions between the modules may be modulated by sensory feedback (Lacquaniti et al., 2012b) dictating the "pace" of the movement. The present results are the first to date demonstrating a correlation of the modular organization of a locomotor task to global performance parameters. The timing for activation patterns during locomotion may define the type of task (walking, running, cutting, etc.) and also its efficiency while the lower limb is interacting with the surface. The association indexes found $\left(r^{2}=0.43\right.$ and 0.45$)$ suggest that the efficiency during changes in direction is partially explicable by the activation timing of motor modules. It is likely that other aspects of motor performance such as motor skill, muscle strength, etc. also have to be considered as part of a successful performance during cutting manoeuvres.

\section{CONCLUSION}

The neural control of complex locomotion tasks, such as cutting manoeuvres, can be described by a lowdimensional set of motor modules which are similar across subjects. These modules are controlled in an impulsive manner, in relation to the biomechanical goals of the task (impact absorption, propulsion). The relative timing for switching between modules may partially determine the external work production to move the CoM. Thus, the optimal progression during locomotor tasks can be partially related to the correct timing to activate muscle weightings. Complex and fast movements can be better performed by refining the relative timing of the activation of motor modules related to specific biomechanical goals.

\section{AUTHORS CONTRIBUTION}

A.O., P.B.S., U.K. and D.F. designed the experiment. A.O. and P.B.S. performed the experiments. A.O., P.B.S., M.E.L., U.K. and D.F. analysed and interpreted the data. A.O., U.K. and D.F. drafted the manuscript and all authors approved the final version. Experiments were performed at Aalborg University.

Acknowledgements-The authors are grateful to Leonardo Gizzi for his comments on the drafts of this manuscript. The authors also would like to thank the Coordenação de Aperfeiçoamento de Pessoal de Nivel Superior (CAPES) for financial support to Anderson S. C. Oliveira for his PhD studies at Aalborg University (process 0293-09-1) and the EU project BETTER (contract 247935) (DF).

\section{REFERENCES}

Andersen MS, Damsgaard M, MacWilliams B, Rasmussen J (2010) A computationally efficient optimisation-based method for parameter identification of kinematically determinate and overdeterminate biomechanical systems. Comput Methods Biomech Biomed Eng 13:171-183.

Besier TF, Lloyd DG, Ackland TR (2003) Muscle activation strategies at the knee during running and cutting maneuvers. Med Sci Sports Exerc 35:119-127.

Cappellini G, Ivanenko YP, Poppele RE, Lacquaniti F (2006) Motor patterns in human walking and running. J Neurophysiol 95:3426-3437.

Cavagna GA (1975) Force platforms as ergometers. J Appl Physiol 39:174-179.

Clark DJ, Ting LH, Zajac FE, Neptune RR, Kautz SA (2010) Merging of healthy motor modules predicts reduced locomotor performance and muscle coordination complexity post-stroke. J Neurophysiol 103:844-857.

d'Avella A, Bizzi E (2005) Shared and specific muscle synergies in natural motor behaviors. Proc Natl Acad Sci U S A 102:3076-3081. 
d'Avella A, Saltiel P, Bizzi E (2003) Combinations of muscle synergies in the construction of a natural motor behavior. Nat Neurosci 6:300-308.

Dempsey AR, Lloyd DG, Elliott BC, Steele JR, Munro BJ, Russo KA (2007) The effect of technique change on knee loads during sidestep cutting. Med Sci Sports Exerc 39:1765-1773.

Gabriel DA, Kamen G, Frost G (2006) Neural adaptations to resistive exercise: mechanisms and recommendations for training practices. Sports Med 36:133-149.

Gizzi L, Nielsen JF, Felici F, Ivanenko YP, Farina D (2011) Impulses of activation but not motor modules are preserved in the locomotion of subacute stroke patients. J Neurophysiol 106:202-210.

Hamner SR, Seth A, Delp SL (2010) Muscle contributions to propulsion and support during running. J Biomech 43: 2709-2716.

Hermens HJ, Freriks B, Disselhorst-Klug C, Rau G (2000) Development of recommendations for SEMG sensors and sensors placement procedures. J Electromyogr Kinesiol 10:361-374.

Ivanenko YP, Poppele RE, Lacquaniti F (2004) Five basic muscle activation patterns account for muscle activity during human locomotion. J Physiol (Lond) 556:267-282.

Ivanenko YP, Cappellini G, Dominici N, Poppele RE, Lacquaniti F (2005) Coordination of locomotion with voluntary movements in humans. J Neurosci 25:7238-7253.

Ivanenko YP, Poppele RE, Lacquaniti F (2006) Motor control programs and walking. Neuroscientist 12:339-348.

Ivanenko Y, Cappellini G, Poppele R, Lacquaniti F (2008) Spatiotemporal organization of $\alpha$-motoneuron activity in the human spinal cord during different gaits and gait transitions. Eur J Neurosci 27:3351-3368.

Jindrich DL, Besier TF, Lloyd DG (2006) A hypothesis for the function of braking forces during running turns. J Biomech 39:1611-1620.

Lacquaniti F, Ivanenko YP, Zago M (2012a) Development of human locomotion. Curr Opin Neurobiol 22:822-828.

Lacquaniti F, Ivanenko YP, Zago M (2012b) Patterned control of human locomotion. J Physiol 590:2189-2199.

Lee DD, Seung HS (1999) Learning the parts of objects by nonnegative matrix factorization. Nature 401:788-791.

Muceli S, Boye AT, d'Avella A, Farina D (2010) Identifying representative synergy matrices for describing muscular activation patterns during multidirectional reaching in the horizontal plane. J Neurophysiol 103:1532-1542.

Neptune RR, Wright IANC, Van den Bogert AJ (1999) Muscle coordination and function during cutting movements. Med Sci Sports Exerc 31:294-302.

Rand MK, Ohtsuki T (2000) EMG analysis of lower limb muscles in humans during quick change in running directions. Gait Posture 12:169-183.

Saibene F, Minetti AE (2003) Biomechanical and physiological aspects of legged locomotion in humans. Eur J Appl Physiol 88:297-316.

Sigward S, Powers CM (2006) The influence of experience on knee mechanics during side-step cutting in females. Clin Biomech 21:740-747.

Torres-Oviedo G, Ting LH (2007) Muscle synergies characterizing human postural responses. J Neurophysiol 98:2144-2156.

van Doornik J, Sinkjaer T (2007) Robotic platform for human gait analysis. IEEE Trans Biomed Eng 54:1696-1702. 